\title{
Cateterización venosa femoral: ¿realmente hay que evitarla?
}

\author{
L. Lorente ${ }^{a, *}$ y C. León ${ }^{b}$ \\ aUnidad de Cuidados Intensivos, Hospital Universitario de Canarias, La Laguna, Tenerife, España \\ bUnidad de Cuidados Intensivos, Hospital Universitario de Valme, Sevilla, España
}

Recibido el 8 de febrero de 2009; aceptado el 19 de marzo de 2009

Disponible en Internet el 4 de octubre de 2009

\section{PALABRAS CLAVE \\ Catéter; \\ Bacteriemia; \\ Femoral; \\ Impregnados}

\section{KEYWORDS \\ Catheter; \\ Bacteremia; \\ Femoral; \\ Impregnated}

\begin{abstract}
Resumen
En las guidelines para la prevención de la bacteriemia relacionada con catéter venoso central (BCVC) de los Centers for Disease Control and Prevention (CDC) de 2002, de la Sociedad Española de Medicina Intensiva, Crítica y Unidades Coronarias/ Sociedad Española de Enfermedades Infecciosas y Microbiología Clínica (SEMICYUC/SEIMC) de 2004, y en las recientemente publicadas guidelines de la Society for Healthcare Epidemiology of America/Infectious Diseases Society of America (SHEA/IDSA) de 2008 se recomienda utilizar preferentemente la vena subclavia y evitar la femoral, y considerar el uso de CVC impregnados en antisépticos o antimicrobianos en unidades hospitalarias o grupos de pacientes que tienen una alta incidencia de BCVC.

A la hora de la implantación de estas guidelines podrían plantearse dos preguntas: 1) ¿el abuso de la vena subclavia y el desuso de la femoral podrían conllevar una disminución de la incidencia de BCVC pero un aumento de la tasa de complicaciones mecánicas como neumotórax o hemotórax?, y 2) ¿no se podrían utilizar catéteres impregnados en antimicrobianos para prevenir la BCVC cuando se utilice la vena femoral? (C) 2009 Elsevier España, S.L. y SEMICYUC. Todos los derechos reservados.
\end{abstract}

Femoral venous catheterization. Does it really need to be avoided?

\begin{abstract}
The guidelines to prevent central venous catheter related bloodstream infections (CVCBSI) of the Centers for Disease Control and Prevention (CDC) of 2002, Sociedad Española de Medicina Intensiva, Crítica y Unidades Coronarias/ Sociedad Española de Enfermedades Infecciosas y Microbiología Clínica (SEMICYUC/SEIMC) of 2004, and the recently published guidelines of the Society for Healthcare Epidemiology of America/Infectious Diseases Society of America (SHEA(IDSA) of 2008 have recommended using the subclavian vein and avoiding the use of the femoral vein. They also recommend considering the use of antiseptic- or antimicrobial-impregnated CVCs for hospital units or groups of patients with a high incidence of CVCBSI.
\end{abstract}

\footnotetext{
*Autor para correspondencia.

Correo electrónico: lorentemartin@msn.com (L. Lorente).
} 
When implementing these guidelines, two questions could be asked: 1) Could the abuse of the subclavian vein and avoiding the use of the femoral vein imply a decrease in the incidence of CVCBSI, but an increase in the rate of mechanical complications as pneumothorax and/or hemothorax? 2) Couldn't antimicrobial-impregnated CVCs be used to prevent CVCBSI when the femoral venous access is used?

(c) 2009 Elsevier España, S.L. and SEMICYUC. All rights reserved.

\section{Introducción}

La cateterización venosa central tiene varias indicaciones, como la existencia de malos accesos venosos periféricos, monitorizar la presión venosa central, la administración de fármacos que producen flebitis al administrarse a través de una vena periférica, la colocación de marcapasos endocavitarios, la realización de hemodiálisis y la administración de nutrición parenteral.

La proporción de pacientes que ingresan en los hospitales y a los que se les realiza la inserción de un catéter venoso central (CVC) se ha incrementado en los últimos años ${ }^{1}$. La utilización de un CVC es muy superior entre los pacientes críticos, y el $78 \%$ de los pacientes ingresados en una unidad de cuidados intensivos ( $\mathrm{UCI}$ ) europea precisa algún $\mathrm{CVC}^{2}$.

La canalización de catéteres intravasculares conlleva diferentes riesgos: infección, neumotórax, hemotórax, embolia gaseosa, hematoma local, trombosis. La bacteriemia relacionada con catéter venoso central (BCVC) es una complicación que aparece en el $1-15 \%$ de los $\mathrm{CVC}^{3-23}$, y conlleva un incremento de la morbimortalidad y de los costes asistenciales ${ }^{24-30}$. El aumento de los costes asistenciales se debe a la necesidad de tratamiento antimicrobiano, de pruebas complementarias para su diagnóstico y a la prolongación de la estancia hospitalaria.

En las guidelines de los Centers for Disease Control and Prevention (CDC) de $1996^{31}$ y de $2002^{32}$ se recomienda la inserción de un CVC por vía subclavia mejor que por vía femoral o yugular para minimizar el riesgo de infección.

En el año 2004 se publicó la conferencia de consenso para la prevención y el tratamiento de las infecciones relacionadas con catéteres intravasculares de corta permanencia en adultos, elaborada por el Grupo de Trabajo de Enfermedades Infecciosas (GTEI) de la Sociedad Española de Medicina Intensiva, Crítica y Unidades Coronarias (SEMICYUC) y el Grupo de Estudio de Infección Hospitalaria (GEIH) de la Sociedad Española de Enfermedades Infecciosas y Microbiología Clínica (SEIMC), celebrada en Toledo en enero del año $2002^{33}$. En esa conferencia de consenso se recomendaba el acceso por vena subclavia, y se proponían los accesos yugular, femoral y axilar como alternativas válidas, pero con mayor riesgo de infección.

Posteriormente, se publicó en 2006 la experiencia de Provonost et al, que desarrollaron un programa en $103 \mathrm{UCl}$ del estado de Michigan que fue capaz de disminuir la incidencia de $\mathrm{BCVC}^{34}$. Este programa se basaba en las siguientes recomendaciones: implementación de una lista de objetivos que se revisaba diariamente; elección en cada $\mathrm{UCl}$ de un líder de la vigilancia de la infección entre el personal médico y otro entre el personal de enfermería; instauración de 5 medidas (higiene de manos, asepsia máxima durante la inserción, asepsia de la piel del punto de entrada con clorhexidina al $2 \%$, evitar el acceso femoral y retirar los catéteres cuando sean innecesarios); utilización de un carro de vía central, y monitorización mensual de las tasas de BCVC. Con este programa se consiguió disminuir la mediana de la densidad de incidencia de BCVC de 2,7 a 0/ 1.000 días de CVC y la media de 7,7 a 1,4/1.000 días de CVC.

También en el año 2006, se publicó la experiencia de Warren et al en $13 \mathrm{UCl}$ de 6 hospitales americanos que tenían una densidad de incidencia superior a la media del National Nosocomial Infection Surveillance System (NNISS) que solamente mediante un programa educativo consiguió reducir la densidad de incidencia de $\mathrm{BCVC}^{35}$. Este programa educativo consistió en actualización de los protocolos escritos, lecturas didácticas, introducción de las recomendaciones basadas en la evidencia en la inserción y el cuidado del catéter, y distribución de un examen preimplantación y postimplantación del programa educativo. Este programa consiguió disminuir la densidad de incidencia de BCVC de 11,2 a 8,9 episodios por 1.000 días de catéter (riesgo relativo $=0,79$; intervalo de confianza (IC) del $95 \%=0,67-0,93$ ) de catéter.

En las recientemente publicadas guidelines de la Society for Healthcare Epidemiology of America/Infectious Diseases Society of America (SHEA/IDSA) para la prevención de la $B C V C$, de 2008, se realizan las siguientes recomendaciones $^{36}$ : a) evitar la femoral porque está asociada a un mayor riesgo de infección y de trombosis venosa profunda, y b) en varios estudios no aleatorizados se ha encontrado una mayor incidencia de BCVC en la vía yugular que en la vía subclavia, pero los riesgos y beneficios en relación con las potenciales complicaciones infecciosas y no infecciosas deben considerarse en cada paciente antes de decidir el sitio venoso.

Actualmente se encuentra en marcha el proyecto Bacteriemia Zero de prevención de las bacteriemias relacionadas con catéteres venosos centrales en las $\mathrm{UCl}$ españolas. Este proyecto se ha puesto en marcha con la colaboración de la Alianza Mundial para la Seguridad del Paciente de la Organización Mundial de la Salud (OMS), la Agencia de Calidad del Ministerio de Sanidad y Consumo (MSC) y la Sociedad Española de Medicina Intensiva Crítica y Unidades Coronarias (SEMICYUC). España es el primer país en seguir la experiencia del estado de Michigan realizada por el Dr. Provonost, y la intervención cuenta con dos actividades complementarias e igualmente importantes: 1) la intervención estandarizada de prevención de BCVC en relación con la inserción y manejo de los catéteres (STOP$B R C)$, cuyo objetivo consiste en disminuir la densidad de incidencia nacional de BCVC por debajo de 4 episodios por 1.000 días de catéter, y 2) implantar el Plan de Seguridad Integral (PSI) que es un marco estratégico para promover y fortalecer la cultura de la seguridad en el trabajo diario en las UCl del Sistema Nacional de Salud. Para conseguir el 
objetivo de la prevención de las BCVC se ha propuesto la implantación de las siguientes 6 medidas: a) higiene adecuada de manos; b) uso de clorhexidina en la preparación de la piel; c) uso de medidas de barrera total durante la inserción del CVC; d) preferencia de la vena subclavia como lugar de inserción, pero teniendo en cuenta otros factores como la posibilidad de complicaciones no infecciosas y la capacidad del facultativo en la inserción de catéteres; e) retirada de CVC innecesarios, y f) manejo higiénico de los catéteres. Es importante recalcar el hecho de que en este programa se va a evaluar también la tasa de neumotórax relacionados en la inserción de vías venosas centrales, aspecto que no se había contemplado en las experiencias previas (Provonost et $\mathrm{al}^{34}$, Warren et $\mathrm{a}^{35}$ ).

A la hora de la implantación de todas estas guidelines, con el mensaje de utilizar preferentemente la vena subclavia y evitar la femoral, podrían plantearse dos preguntas: 1) ¿el abuso de la vena subclavia y el desuso de la femoral podrían conllevar una disminución de la incidencia de BCVC pero un aumento de la tasa de complicaciones mecánicas como neumotórax o hemotórax?, y 2) ¿no se podrían utilizar catéteres impregnados en antimicrobianos para prevenir la BCVC cuando se utilice la vena femoral?

\section{Complicaciones infecciosas}

Por el momento, no existe ningún estudio aleatorizado que haya comparado la tasa de BCVC según los diferentes accesos vasculares (subclavia, yugular y femoral).

Los resultados de los estudios apuntan a una menor tasa de colonización de la punta del catéter con la vena subclavia que con la femoral y yugular ${ }^{4-8,10,11,15,21}$, pero los resultados no son concordantes respecto a la comparación entre femoral y yugular. $Y$ en un único estudio se ha encontrado una mayor incidencia de BCVC en femoral que en subclavia y yugular ${ }^{12}$.

En varios estudios se ha encontrado una mayor incidencia de colonización de la punta del catéter en femoral que en subclavia y yugular ${ }^{4,7,10}$ o en femoral que en subclavia ${ }^{11,15}$. Sin embargo, en otros estudios se ha encontrado una mayor incidencia de colonización de la punta del catéter en yugular que en femoral y subclavia ${ }^{5,6,8}$ o en yugular que en subclavia ${ }^{15,21}$. Pero la gran limitación de todos los estudios es que son obervacionales, salvo el de Merrer et al, en el que se distribuye al azar la canalización de femoral o subclavia ${ }^{11}$.

\section{Complicaciones mecánicas de los catéteres venosos centrales}

Además de las complicaciones infecciosas, la canalización de una vía central también conlleva el riesgo de complicaciones mecánicas ${ }^{9,11,19,22,37-64}$.

Dentro de las complicaciones mecánicas de la cateterización de la vena subclavia se encuentra el neumotórax, en el $0-6 \%$, el hemotórax, en el $0-1,4 \%$, la embolia gaseosa, en el $0,7-0,9 \%$, la punción arterial, en el $0-7,8 \%$ y la trombosis venosa, en el $0-50 \%$ (tablas $1,3,4$ ).

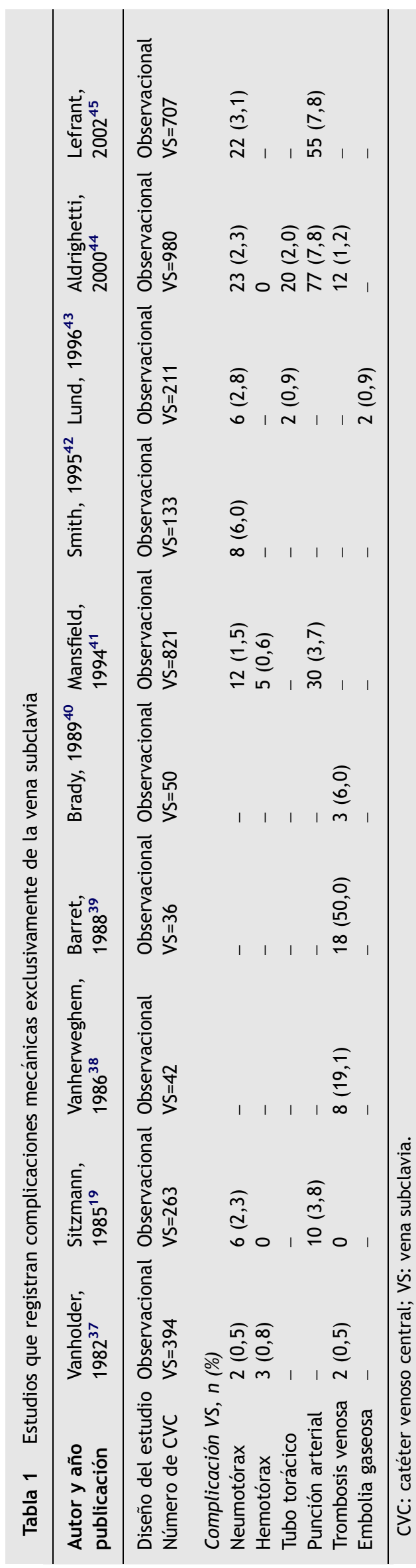


Tabla 2 Estudios que registran complicaciones mecánicas exclusivamente de la vena yugular

\begin{tabular}{lllll}
\hline Autor y año publicación & Canaud, 1986 & Donnelly, 1988 & Cappello, 1989 & Gibson, 199149 \\
\hline $\begin{array}{l}\text { Diseño del estudio } \\
\text { Número de CVC }\end{array}$ & $\begin{array}{l}\text { Observacional } \\
\text { VY=138 }\end{array}$ & Observacional & Observacional & Observacional \\
VY=47 & & & -107 \\
Complicación VY, $n(\%)$ & & & - & - \\
Neumotórax & - & - & - & - \\
Hemotórax & - & - & - & - \\
Tubo torácico & - & - & - & - \\
Punción arterial & - & - & $14(13,1)$ & - \\
Trombosis venosa & $2(1,4)$ & $2(4,2)$ & - & - \\
Embolia gaseosa & $1(0,7)$ & - & & \\
\hline
\end{tabular}

CVC: catéter venoso central; VY: vena yugular.

Dentro de las complicaciones mecánicas de la cateterización de la vena yugular se encuentra el neumotórax, en el 0-3\%, el hemotórax, en el 0-1,5\%, la embolia gaseosa, en el 0,4-0,7\%, la punción arterial, en el 0-9,4\% y la trombosis venosa, en el $0-13,1 \%$ (tablas 2-4).

La aparición de neumotórax o hemotórax en la canalización de la vena yugular o subclavia puede conllevar la necesidad de la colocación de un tubo torácico, el desarrollo de un importante deterioro respiratorio o hemodinámico e incluso el fallecimiento del paciente. Aunque existen escasos datos publicados sobre estas complicaciones, en algún estudio se ha reportado una tasa de colocación de tubo torácico del $0,9-2 \%{ }^{43,44}$.

Otra posible complicación que puede aparecer durante la canalización de la vena yugular es la punción y canalización de la arteria carótida, que puede conllevar la aparición de ictus isquémicos, que podrían ser graves, como se reportó en el estudio de Schummer ${ }^{64}$. También se han descrito taponamientos cardíacos durante la implantación de CVC por subclavia o yugular ${ }^{65}$.

Dentro de las complicaciones mecánicas de la cateterización de la vena femoral se encuentra la punción arterial, en el $7,1-15 \%$ y la trombosis venosa, en el 2,1-17,2\% (tabla 4). Por lo tanto, la incidencia de punción arterial es mayor con la femoral, pero el riesgo de trombosis es menor que en la subclavia, y lógicamente no existe el riesgo de neumotórax, de hemotórax ni de embolia gaseosa.

\section{Catéteres venosos centrales impregnados en antimicrobianos}

Aunque en varios metaanálisis se ha evidenciado una disminución de la BCVC con el uso de un CVC impregnado con antimicrobiano o antiséptico, su uso no se ha generalizado debido a que son más costosos que los catéteres estándares $^{66-69}$.

Las recomendaciones de las guidelines de los CDC de 2002 respecto a la utilización de CVC impregnados en antimicrobianos son las siguientes ${ }^{32}$ : usar un CVC impregnado con antimicrobiano o antiséptico en adultos en quienes se espera mantener el catéter durante más de 5 días, después de implementar una comprensiva estrategia para reducir las tasas de BCVC y las tasas se mantiene por encima de los objetivos propuestos por la institución. Esta estrategia debe incluir los siguientes 3 componentes: educación del personal que inserta y mantiene los catéteres, utilizar máximas precauciones de barrera y preparar la zona cutánea con clorhexidina al $2 \%$.

En las recomendaciones de las guidelines de la SEIMCSEMICYUC de 2004 se concluye que la eficacia de los CVC impregnados con antimicrobiano o antiséptico para la prevención de infecciones está siendo evaluada todavía ${ }^{33}$. Aunque no es posible hacer recomendaciones definitivas para su uso, la evidencia existente permite aconsejar su uso en aquellos centros en los que las tasas de infección sean superiores a los estándares establecidos. En estos centros se deben optimizar todas las precauciones en la inserción y posterior cuidado de los catéteres, así como reactivar los programas de educación.

En las recientemente publicadas guidelines de la SHEA/ IDSA para la prevención de la BCVC, de 2008, se propone considerar el uso de CVC impregnados en antisépticos o en antimicrobianos en las siguientes circunstancias ${ }^{36}:$ a) unidades hospitalarias o grupos de pacientes que tienen una incidencia de BCVC superior a la estimada, a pesar de la cumplimentación de las medidas básicas para la prevención de BCVC; b) pacientes que tienen limitaciones de accesos venosos y antecedentes personales de BCVC recurrente, y c) pacientes con alto riesgo de secuelas debido a BCVC (p. ej., pacientes con implantación reciente de dispositivos intravasculares como válvulas cardíacas o prótesis aórticas).

En el reciente metaanálisis publicado por estudio de Casey et al, se ha observado que los catéteres impregnados en clorhexidina-sulfadizina argéntica reducen la colonización de los CVC (odds ratio: 0,51; IC del 95\%: $0,42-0,61$ ) y BCVC (odds ratio: 0,68 ; IC del 95\%: 0,47-0,98), y que los catéteres impregnados en rifampicina-minociclina disminuyen la colonización de CVC (odds ratio: 0,39 ; IC del 95\%: 0,27-0,55) y de BCVC (odds ratio: 0,29; IC del 95\%: $0,16-0,52)^{69}$.

Posiblemente, el mayor impedimento para la utilización de los CVC impregnados en antimicrobianos sea su coste, pero se podría optimizar su relación coste-beneficio si se utilizara en situaciones de mayor riesgo de BCVC, como en los accesos venosos en sitio femoral. En un estudio retrospectivo de los pacientes que ingresaron en la $\mathrm{UCl}$ y recibieron un CVC en vía femoral o en el acceso central de la vena yugular interna encontramos una menor incidencia de BCVC con la utilización de CVC impregnados en rifampicina- 


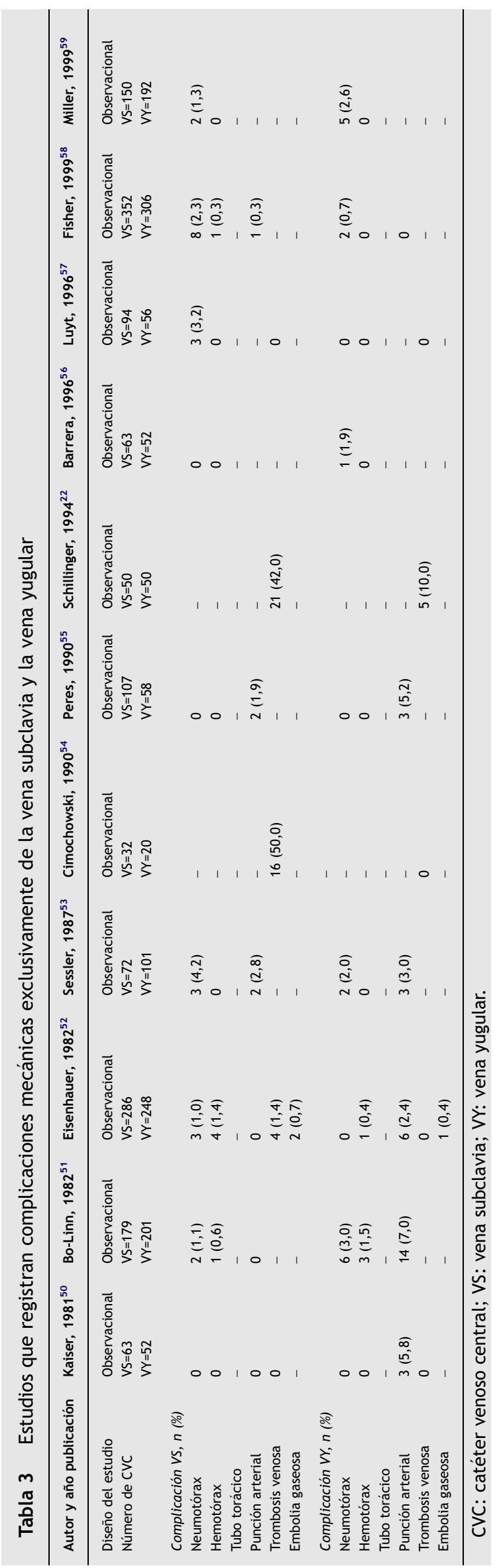

miconazol ${ }^{17}$. Hubo una menor incidencia de BCVC entre los 73 catéteres impregnados en rifampicina-miconazol respecto a los 111 CVC no impregnados en el acceso femoral ( 0 versus 8,62 por 1.000 catéter-día, odds ratio: 0,13; IC del 95\%: 0,00-0,86; $p=0,03$ ). También encontramos una menor incidencia de BCVC en los 114 CVC impregnados que en los 127 CVC no impregnados utilizados en el acceso central de la vena yugular interna ( 0 versus 4,93 por 1.000 catéter-día, odds ratio: 0,13; IC del 95\%: 0,00-0,93; p=0,04). La incidencia de BCVC en vena femoral y en el acceso central de la vena yugular interna con CVC impregnados no fue diferente a la incidencia en vena subclavia con CVC no impregnados. En otros dos estudios en los que se incluyeron exclusivamente enfermos críticos no se encontró un descenso de la BCVC con la utilización de CVC impregnados en rifampicina-minociclina ${ }^{70,71}$. Aunque en el estudio de León et al, el acceso femoral no fue utilizado ${ }^{70}$, y en el estudio de Fraenkel et al el acceso femoral fue utilizado sólo en el $12 \%$ de los $\operatorname{casos}^{71}$. También en el estudio de Chatzinikolau et al en catéteres utilizados para hemodiálisis se encontró una menor incidencia de BCVC con catéteres impregnados en rifampicina-minocidina (C-RM) que con Catéteres estándar (C-S) $(0 / 66 \text { versus } 7 / 64(10,9 \%) ; p=0,006)^{72}$.

En nuestra experiencia hemos encontrado las siguientes diferencias en la incidencia de BCVC según el sitio utilizado: a) una mayor incidencia de BCVC con la utilización de la vena femoral que con el uso de la vena subclavia ${ }^{12}$ y yugular ${ }^{73}$; b) una superior incidencia de BCVC con el acceso central de la vena yugular interna que con el acceso posterior $^{74}$, posiblemente debido a que se coloca a los pacientes críticos en la posición de semidecúbito para evitar la neumonía asociada a ventilación mecánica, y entonces las secrecciones orofaríngeas alcanzan por la acción de la gravedad más fácilmente el acceso central que el posterior; c) una mayor incidencia de BCVC con la utilización de la vena yugular por acceso central que con el uso de la vena subclavia $^{12}$; d) no hay diferencias en la incidencia de BCVC entre la vena subclavia y el acceso posterior de la vena yugular interna ${ }^{75}$, y e) como indicábamos previamente, una menor incidencia de BCVC con los CVC impregnados en rifampicina-miconazol que con los CVC no impregnados en la vía femoral y el acceso central de la vena yugular interna ${ }^{17}$, además de una diferencia en la etiología de los microorganismos causantes de BCVC según el sitio de la bacteriemia $^{76-78}$.

Finalmente, creemos en la utilidad de la implantación de un paquete de medidas para el control de las infecciones nosocomiales, y para mejorar la seguridad de los pacientes $^{79}$.

\section{Conclusión}

La elección del acceso venoso debería realizarse en función del riesgo de las posibles complicaciones infecciosas y no infecciosas, y en función de la capacidad del personal implicado en la canalización del acceso venoso. Se podría tener en cuenta la utilización de los catéteres impregnados en antimicrobianos en pacientes con mayor riesgo de desarrollar complicaciones infecciosas como canalización de vena femoral, pacientes imunodeprimidos o alteraciones de la integridad cutánea. 
Tabla 4 Estudios que registran complicaciones mecánicas de la vena femoral

\begin{tabular}{|c|c|c|c|c|c|c|c|}
\hline $\begin{array}{l}\text { Autor y año } \\
\text { publicación }\end{array}$ & $\begin{array}{l}\text { Williams, } \\
1991^{60}\end{array}$ & $\begin{array}{l}\text { Durbec, } \\
1997^{9}\end{array}$ & Timsit, $1999^{61}$ & $\begin{array}{l}\text { Merrer, } \\
2001^{11}\end{array}$ & $\begin{array}{l}\text { lovino, } \\
2001^{62}\end{array}$ & $\begin{array}{l}\text { Eisen, } \\
2006^{63}\end{array}$ & $\begin{array}{l}\text { Schummer, } \\
2007^{64}\end{array}$ \\
\hline $\begin{array}{l}\text { Diseño del } \\
\text { estudio }\end{array}$ & Observacional & Observacional & $\begin{array}{l}\text { Aleatoriza tunelizado } \\
\text { vs. no tunelizado }\end{array}$ & $\begin{array}{l}\text { Aleatoriza } \\
\text { VS vs. VF }\end{array}$ & Observacional & Observacional & Observacional \\
\hline Número de & VS=0 & $\mathrm{VS}=0$ & $\mathrm{VS}=0$ & VS=144 & $V S=555$ & $V S=218$ & VS=729 \\
\hline \multirow[t]{2}{*}{ CVC } & $V Y=0$ & $V Y=0$ & $V Y=0$ & $V Y=0$ & $V Y=1.116$ & $V Y=40$ & $V Y=339$ \\
\hline & $V F=150$ & $V F=80$ & $V F=336$ & $V F=145$ & $V F=619$ & $V F=127$ & $V F=28$ \\
\hline \multicolumn{8}{|c|}{ Complicación VS, n (\%) } \\
\hline Neumotórax & N/A & N/A & N/A & $4(2,8)$ & $17(3,1)$ & $5(2,3)$ & $3(0,4)$ \\
\hline Hemotórax & N/A & $\mathrm{N} / \mathrm{A}$ & N/A & - & - & $1(0,5)$ & 0 \\
\hline Tubo torácico & N/A & N/A & N/A & - & - & - & - \\
\hline $\begin{array}{l}\text { Punción } \\
\text { arterial }\end{array}$ & N/A & N/A & N/A & $7(4,9)$ & $15(2,7)$ & $7(3,2)$ & $7(1,0)$ \\
\hline $\begin{array}{l}\text { Trombosis } \\
\text { venosa }\end{array}$ & N/A & $\mathrm{N} / \mathrm{A}$ & N/A & $2(1,4)$ & - & - & - \\
\hline $\begin{array}{l}\text { Embolia } \\
\text { gaseosa }\end{array}$ & N/A & N/A & N/A & - & - & - & - \\
\hline \multicolumn{8}{|c|}{ Complicación VY, n (\%) } \\
\hline Neumotórax & N/A & $\mathrm{N} / \mathrm{A}$ & N/A & N/A & 0 & 0 & $4(1,2)$ \\
\hline Hemotórax & N/A & $\mathrm{N} / \mathrm{A}$ & N/A & N/A & - & 0 & $1(0,3)$ \\
\hline Tubo torácico & N/A & $\mathrm{N} / \mathrm{A}$ & N/A & N/A & - & - & - \\
\hline $\begin{array}{l}\text { Punción } \\
\text { arterial }\end{array}$ & N/A & $\mathrm{N} / \mathrm{A}$ & N/A & N/A & $11(1,0)$ & $2(5,0)$ & $32(9,4)$ \\
\hline $\begin{array}{l}\text { Trombosis } \\
\text { venosa }\end{array}$ & N/A & $\mathrm{N} / \mathrm{A}$ & N/A & N/A & - & - & - \\
\hline $\begin{array}{l}\text { Embolia } \\
\text { gaseosa }\end{array}$ & N/A & N/A & N/A & N/A & - & - & - \\
\hline \multicolumn{8}{|c|}{ Complicación VF, n (\%) } \\
\hline Neumotórax & N/A & $\mathrm{N} / \mathrm{A}$ & N/A & N/A & $\mathrm{N} / \mathrm{A}$ & N/A & N/A \\
\hline Hemotórax & N/A & $\mathrm{N} / \mathrm{A}$ & N/A & N/A & $\mathrm{N} / \mathrm{A}$ & N/A & N/A \\
\hline Tubo torácico & N/A & $\mathrm{N} / \mathrm{A}$ & $\mathrm{N} / \mathrm{A}$ & N/A & $\mathrm{N} / \mathrm{A}$ & N/A & N/A \\
\hline $\begin{array}{l}\text { Punción } \\
\text { arterial }\end{array}$ & $14(9,3)$ & $12(15,0)$ & $25(7,4)$ & $13(9,0)$ & - & $9(7,1)$ & $2(7,1)$ \\
\hline $\begin{array}{l}\text { Trombosis } \\
\text { venosa }\end{array}$ & - & $6(7,5)$ & $7(2,1)$ & $25(17,2)$ & - & - & - \\
\hline $\begin{array}{l}\text { Embolia } \\
\text { gaseosa }\end{array}$ & - & - & - & - & - & - & - \\
\hline
\end{tabular}

CVC: catéter venoso central; N/A: no aplicable; VF: vena femoral; VS: vena subclavia; VY: vena yugular.

\section{Financiación}

Fondos hospitalariós

\section{Bibliografía}

1. Vaqué J, Rosselló J, Arribas JL, EPINE Working Group. Prevalence of nosocomial infections in Spain: EPINE study 1990-1997. EPINE Working Group. J Hosp Infect. 1999;43:S105-11.

2. Vincent JL, Bihari DJ, Suter PM, Bruining HA, White J, Nicolas $\mathrm{MH}$, the EPIC International Advisory Committee. The prevalence of nosocomial infection in Intensive Care Units in Europe. JAMA. 1995;274:639-44.
3. Bozzetti F, Terno G, Camerini E, Baticci F, Scarpa D, Pupa A. Pathogenesis and redictability of central venous catheter sepsis. Surgery. 1982;91:383-9.

4. Collignon P, Soni N, Pearson I, Sorrell T, Woods P. Sepsis associated with central vein catheters in critically ill patients. Intensive Care Med. 1988;14:227-31.

5. Richet $\mathrm{H}$, Hubert B, Nitemberg $G$, Andremont A, Buu-Hoi A, Ourbak $C$, et al. Prospective multicenter study of vascularcatheter-related complications and risk factors for positive central-catheter culture in intensive care unit patients. J Clin Microbiol. 1990;28:2520-5.

6. Mermel LA, McCormick RD, Springman SR, Maki DG. The pathogenesis and epidemiology of catheter-related infection with pulmonary artery Swan-Ganz catheters: A prospective 
study utilizing molecular subtyping. Am J Med. 1991;91:197S-205S.

7. Kemp L, Burge J, Choban P, Harden J, Mirtallo J, Flancbaum L. The effect of catheter type and site on infection rates in total parenteral nutrition patients. J Parenter Enteral Nutr. 1994;18:71-4.

8. Moro ML, Vigano EF, Cozzi A. Risk factors for central venous catheter-related infections in surgical and intensive care units. The Central Venous Catheter Related Infections Study Group. Infect Control Hosp Epidemiol. 1994;15:253-64.

9. Durbec O, Vivand X, Potie F, Vialet R, Albanese J, Martin C. A prospective evaluation of the use of femoral venous catheters in critically ill adults. Crit Care Med. 1997;25:1986-9.

10. Goetz AM, Wagner MM, Miller JM, Muder RR. Risk of infection due to central venous catheters: Effect of site of placement and catheter type. Infect Control Hosp Epidemiol. 1986;19:842-5.

11. Merrer J, De Jonghe B, Golliot F, Lefrant JY, Raffy B, Barre E. Complications of femoral and subclavian venous catheterization in critically ill patients. JAMA. 2001;286:700-7.

12. Lorente L, Henry C, Martin MM, Jiménez A, Mora ML. Central venous catheter-related infection in a prospective and observational study of 2595 catheters. Critical Care. 2005;9:R631-5.

13. Deshpande KS, Hatem C, Ulrich HL, Currie BP, Aldrich TK, BryanBrown CW, et al. The incidence of infectious complications of central venous catheters at the subclavian, internal jugular, and femoral sites in an intensive care unit population. Crit Care Med. 2005;33:13-20.

14. Nagashima G, Kikuchi T, Tsuyuzaki H, Kawano R, Tanaka H, Nemoto $\mathrm{H}$, et al. To reduce catheter-related bloodstream infections: Is the subclavian route better than the jugular route for central venous catheterization?. J Infect Chemother. 2006;12:363-5.

15. Gowardman JR, Robertson IK, Parkes S, Rickard CM. Influence of insertion site on central venous catheter colonization and bloodstream infection rates. Intensive Care Med. 2008;34:1038-45.

16. Garnacho-Montero J, Aldabó-Pallás T, Palomar-Martínez M, Vallés J, Almirante B, Garcés R, et al. Risk factors and prognosis of catheter-related bloodstream infection in critically ill patients: A multicenter study. Intensive Care Med. 2008;34:2185-93.

17. Lorente $L$, Lecuona $M$, Ramos MJ, Jiménez A, Mora ML, Sierra A. The use of rifampicin-miconazole-impregnated catheters reduces the incidence of femoral and jugular catheter-related bacteremia. Clin Infect Dis. 2008;47:1171-5.

18. Pinilla JC, Ross DC, Martin T, Crump H. Study of the incidence of intravascular catheter infection and associated septicaemia in critically ill patients. Crit Care Med. 1983;11:21-5.

19. Sitzmann JV, Townsend TR, Siler MC, Bartlett JG. Septic and technical complications of central venous catheterization. A prospective study of 200 consecutive patients. Ann Surg. 1985;202:766-70.

20. Damen J, Verhoef J, Bolton DT, Middleton NG, Van der Tweer I, Jonge KD. Microbiologic risk of invasive hemodynamic monitoring in patients undergoing open-heart operation. Crit Care Med. 1985;13:548-55.

21. Brun-Buisson C, Abrouk F, Legrand $P$, Huet $Y$, Larabi S, Rapin $M$. Diagnosis of central venous catheter-related sepsis. Critical level of quantitative tip cultures. Arch Intern Med. 1987;147:873-7.

22. Schillinger F, Schillinger D, Montagnac R, Milcent T. Central venous stenosis in hemodialysis: Comparative angiographic study of subclavian and internal jugular access. Nephrologie. 1994;15:129-31.

23. Álvarez-Lerma F, Palomar M, Olaechea P, Otal JJ, Insausti J, Cerdá E, Grupo de Estudio de Vigilacia de Infección Nosocomial en UCl. National Study of Control of Nosocomial Infection in
Intensive Care Units. Evolutive report of the years 2003-2005. Med Intensiva. 2007;31:6-17.

24. Spengler RF, Greenough WB. Hospital costs and mortality attributed to nosocomial bacteremias. JAMA. 1978;240:2455-8.

25. Haley RW, Schaberg DR, Crossley KB, Von Allmen SD, McGowan JE. Extra charges and prolongation of hospitalization attributable to nosocomial infections: A prospective interhospital comparison. Am J Med. 1981;70:51-8.

26. Smith RL, Meixler SM, Simberkoff MS. Excess mortality in critically ill patients with nosocomial bloodstream infections. Chest. 1991;100:164-7.

27. Collignon PJ. Intravascular catheter associated sepsis: A common problem. The Australian Study on Intravascular Catheter Associated Sepsis. Med J Aust. 1994;161:374-8.

28. Pittet D, Tarara D, Wenzel R. Nosocomial bloodstream infection in critically ill patients. Excess length of stay, extra costs and attributable mortality. JAMA. 1994;271:1598-601.

29. Laupland KB, Lee H, Gregson DB, Manns BJ. Cost of intensive care unit-acquired bloodstream infections. J Hosp Infect. 2006;63:124-32.

30. Arnow PM, Quimosing EM, Beach M. Consequences of intravascular catheter sepsis. Clin Infect Dis. 1993;16:778-84.

31. Pearson ML. Guideline for prevention of intravascular devicerelated infections. The Hospital Infection Control Practices Advisory Committee. Am J Infect Control. 1996;24:262-93.

32. O'Grady NP, Alexander M, Dellinger EP, Gerberding JL, Heard SO, Maki DG, et al. Centers for Disease Control and Prevention. Guidelines for the Prevention of Intravascular Catheter-Related Infections. MMWR. 2002;51:1-29.

33. León C, Ariza J, SEIMC, SEMICYUC. Guidelines for the treatment of short-term intravascular catheter-related infections in adults; SEIMC-SEMICYUC Consensus Conference. Enferm Infecc Microbiol Clin. 2004;22:92-101.

34. Pronovost P, Needham D, Berenholtz S, Sinopoli D, Chu H, Cosgrove $S$, et al. An intervention to decrease catheter-related bloodstream infections in the ICU. $N$ Engl $J$ Med. 2006;355:2725-32.

35. Warren DK, Cosgrove SE, Diekema DJ, Zuccotti G, Climo MW, Bolon MK, et al. Prevention Epicenter Program. A multicenter intervention to prevent catheter-associated bloodstream infections. Infect Control Hosp Epidemiol. 2006;27:662-9.

36. Marschall J, Mermel LA, Classen D, Arias KM, Podgorny K, Anderson DJ, et al. Practice Recommendation of Society for Healthcare Epidemiology of America/Infectious Diseases Society of America (SHEA/IDSA). Strategies to prevent central line-associated bloodstream infections in acute care hospitals. Infect Control Hosp Epidemiol. 2008;29:S22-30.

37. Vanholder R, Lameire N, Verbanck J, Van Rattinghe R, Kunnen $M$, Ringoir S. Complications of subclavian catheter hemodialysis: A 5 year prospective study in 257 consecutive patients. Int J Artif Organs. 1982;5:297-303.

38. Vanherweghem JL, Yassine T, Goldman M, Vandenbosch G, Delcour C, Struyven J, et al. Subclavian vein thrombosis: A frequent complication of subclavian vein cannulation for hemodialysis. Clin Nephrol. 1986;26:235-8.

39. Barrett N, Spencer S, Mclvor J, Brown EA. Subclavian stenosis: A major complication of subclavian dialysis catheters. Nephrol Dial Transplant. 1988;3:423-5.

40. Brady HR, Fitzcharles B, Goldberg H, Huraib S, Richardson T, Simons $M$, et al. Diagnosis and management of subclavian vein thrombosis occurring in association with subclavian cannulation for hemodiálisis. Blood Purif. 1989;7:210-17.

41. Mansfield PF, Hohn DC, Fornage BD, Gregurich MA, Ota DM. Complications and failures of subclavian-vein catheterization. $\mathrm{N}$ Engl J Med. 1994;331:1735-8.

42. Smith HO, DeVictoria CL, Garfinkel D, Anderson P, Goldberg GL, Soeiro $\mathrm{R}$, et al. A prospective randomized comparison of an 
attached silver-impregnated cuff to prevent central venous catheter-associated infection. Gynecol Oncol. 1995;58:92-100.

43. Lund GB, Trerotola SO, Scheel Jr. PF, Savader SJ, Mitchell SE, Venbrux AC, et al. Outcome of tunneled hemodialysis catheters placed by radiologists. Radiology. 1996;198:467-72.

44. Aldrighetti L, Paganelli M, Arru M, Caterini R, Ronzoni M, Ferla G. Complications of blind placement technique in 980 subcutaneous infusion ports. J Vasc Access. 2000;1:28-32.

45. Lefrant JY, Muller L, De La Coussaye JE, Prudhomme M, Ripart J, Gouzes C. Risk factors of failure and immediate complication of subclavian vein catheterization in critically ill patients. Intensive Care Med. 2002;28:1036-41.

46. Canaud B, Beraud JJ, Joyeux H, Mion C. Internal jugular vein cannulation using 2 silastic catheters. A new, simple and safe long-term vascular access for extracorporeal treatment. Nephron. 1986;43:133-8.

47. Donnelly PK, Hoenich NA, Lennard TW, Proud G, Taylor RM. Surgical management of long-term central venous access in uraemic patients. Nephrol Dial Transplant. 1988;3:57-65.

48. Cappello M, De Pauw L, Bastin G, Prospert F, Delcour C, Thaysse $C$, et al. Central venous access for haemodialysis using the Hickman catheter. Nephrol Dial Transplant. 1989;4:988-92.

49. Gibson SP, Mosquera DA, O'Donnell MJ, Odurny A. Streptokinase in the treatment of innominate-vein thrombosis in association with haemodialysis catheter. Preservation of a precious arteriovenous fistula. Nephrol Dial Transplant. 1991;6:206-8.

50. Kaiser CW, Koornick AR, Smith N, Soroff HS. Choice of route for central venous cannulation: Subclavian or internal jugular vein? A prospective randomized study. J Surg Oncol. 1981;17:345-54.

51. Bo-Linn GW, Anderson DJ, Anderson KC, McGoon MD. Percutaneous central venous catheterization performed by medical house officers: A prospective study. Cathet Cardiovasc Diagn. 1982;8:23-9.

52. Eisenhauer ED, Derveloy RJ, Hastings PR. Prospective evaluation of central venous pressure (CVP) catheters in a large citycounty hospital. Ann Surg. 1982;196:560-4.

53. Sessler CN, Glauser FL. Central venous cannulation done by house officers in the intensive care unit: A prospective study. South Med J. 1987;80:1239-42.

54. Cimochowski GE, Worley E, Rutherford WE, Sartain J, Blondin J, Harter H. Superiority of the internal jugular over the subclavian access for temporary dialysis. Nephron. 1990;54:154-61.

55. Peres PW. Positioning central venous catheters - a prospective survey. Anaesth Intensive Care. 1990;18:536-9.

56. Barrera R, Mina B, Huang Y, Groeger JS. Acute complications of central line placement in profoundly thrombocytopenic cancer patients. Cancer. 1996;78:2025-30.

57. Luyt DK, Mathivha LR, Litmanovitch M, Dance MD, Brown JM. Confirmation of the safety of central venous catheterisation in critically ill infants and children-the Baragwanath experience. S Afr Med J. 1996;86:603-6.

58. Fisher NC, Mutimer DJ. Central venous cannulation in patients with liver disease and coagulopathy - a prospective audit. Intensive Care Med. 1999;25:481-5.

59. Miller JA, Singireddy S, Maldjian P, Baker SR. A reevaluation of the radiographically detectable complications of percutaneous venous access lines inserted by four subcutaneous approaches. Am Surg. 1999;65:125-30.

60. Williams JF, Seneff MG, Friedman BC, McGrath BJ, Gregg R, Sunner J, et al. Use of femoral venous catheters in critically ill adults: Prospective study. Crit Care Med. 1991;19:550-3.

61. Timsit JF, Bruneel F, Cheval C, Mamzer MF, Garrouste-Orgeas M, Wolff $M$, et al. Use of tunneled femoral catheters to prevent catheter-related infection. A randomized, controlled trial. Ann Intern Med. 1999;130:729-35.

62. lovino F, Pittiruti M, Buononato M, Lo Schiavo F. Central venous catheterization: Complications of different placements. Ann Chir. 2001;126:1001-6.
63. Eisen LA, Narasimhan M, Berger JS, Mayo PH, Rosen MJ, Schneider RF. Mechanical complications of central venous catheters. J Intensive Care Med. 2006;21:40-6.

64. Schummer W, Schummer C, Rose N, Niesen WD, Sakka SG. Mechanical complications and malpositions of central venous cannulations by experienced operators. A prospective study of 1794 catheterizations in critically ill patients. Intensive Care Med. 2007;33:1055-9.

65. Bagwell CE, Salzberg AM, Sonnino RE, Haynes JH. Potentially lethal complications of central venous catheter placement. J Pediatr Surg. 2000;35:709-13.

66. Veenstra DL, Saint S, Saha S, Lumley T, Sullivan SD. Efficacy of antiseptic-impregnated central venous catheters in preventing catheter-related bloodstream infection: A meta-analysis. JAMA. 1999;281:261-7.

67. Marin MG, Lee JC, Skurnick JH. Prevention of nosocomial bloodstream infections: Effectiveness of antimicrobial-impregnated and heparin-bonded central venous catheters. Crit Care Med. 2000;28:3332-8.

68. Falagas ME, Fragoulis K, Bliziotis IA, Chatzinikolaou I. Rifampicin-impregnated central venous catheters: A meta-analysis of randomized controlled trials. J Antimicrob Chemother. 2007;59:359-69.

69. Casey AL, Mermel LA, Nightingale P, Elliott TS. Antimicrobial central venous catheters in adults: A systematic review and meta-analysis. Lancet Infect Dis. 2008;8:763-76.

70. León C, Ruiz-Santana S, Rello J, De la Torre MV, Vallés J, Álvarez-Lerma F, et al. Cabaña Study Group. Benefits of minocycline and rifampin-impregnated central venous catheters. A prospective, randomized, double-blind, controlled, multicenter trial. Intensive Care Med. 2004;30:1891-9.

71. Fraenkel D, Rickard C, Thomas P, Faoagali J, George N, Ware R. A prospective, randomized trial of rifampicin-minocyclinecoated and silver-platinum-carbon-impregnated central venous catheters. Crit Care Med. 2006;34:668-75.

72. Chatzinikolaou I, Finkel K, Hanna H, Boktour M, Foringer J, Ho T, et al. Antibiotic-coated hemodialysis catheters for the prevention of vascular catheter-related infections: A prospective, randomized study. Am J Med. 2003;115:352-7.

73. Lorente L, Jiménez A, García C, Galván R, Castedo J, Martín $M M$, et al. Catheter-related bacteremia from femoral and central internal jugular venous access. Eur J Clin Microbiol Infect Dis. 2008;27:867-71.

74. Lorente L, Jiménez A, Castedo J, Galván R, García C, Martín $M M$, et al. Internal jugular venous catheter-related bacteremia according to central and posterior accesses. Intensive Care Med. 2007;33:1071-5.

75. Lorente L, Jiménez A, Galván R, García C, Castedo J, Martín $M M$, et al. Equivalence of posterior internal jugular and subclavian accesses in the incidence of central venous catheter related bacteremia. Intensive Care Med. 2007;33:2230-1.

76. Lorente L, Jiménez A, Iribarren JL, Jiménez JJ, Martín MM, Mora ML. The micro-organism responsible for central venous catheter related bloodstream infection depends on catheter site. Intensive Care Med. 2006;32:1449-50.

77. Lorente L, Jiménez A, Santana M, Iribarren JL, Jiménez JJ, Martín MM, et al. Microorganisms responsible for intravascular catheter-related bloodstream infection according to the catheter site. Crit Care Med. 2007;35:2424-7.

78. Lorente L, Jiménez A, Mora ML. Microorganisms responsibles for femoral catheter-related bloodstream infection. Crit Care Med. 2008;36:657-8.

79. Lisboa T, Rello J. Prevention of nosocomial infections: Strategies to improve the safety of the patients in the Intensive Care Unit. Med Intensiva. 2008;32:248-52. 